\title{
Single aggressive and non-aggressive social interactions elicit distinct behavioral patterns to the context in mice
}

\author{
Ariela M. Crestani ${ }^{\mathrm{a}, \mathrm{b}, *}$, Ana C. Cipriano ${ }^{\mathrm{a}, \mathrm{b}, *}$, Ricardo L. Nunes-de-Souza ${ }^{\mathrm{a}, \mathrm{b}, *}$ \\ a Department of Natural Active Principles and Toxicology, School of Pharmaceutical Sciences, São Paulo State University - UNESP, Brazil \\ b Joint Graduate Program in Physiological Sciences, UFSCar/UNESP, Brazil
}

\section{A R T I C L E I N F O}

\section{Keywords:}

Social defeat

Contextual conditioning

Contextual memory

Self-grooming

\begin{abstract}
A B S T R A C T
Aggressive interactions between conspecific animals have been used as a social stressor with ethological characteristics to study how social interactions can modulate animal's behavior. Here, a new protocol based on aggressive and non-aggressive interactions was developed to study how different social interactions can alter the behavioral profile of animals re-exposed to the context in which the interaction occurred. We used factor analysis to trace the behavioral profile of socially defeated and non-defeated mice when they were re-exposed to the apparatus [three interconnected chambers: home chamber, tunnel and surface area]; we also compared the behavior presented before (habituation) and $24 \mathrm{~h}$ after (re-exposure) the non-aggressive or aggressive interactions. A final factor analysis from defeated animals yielded 4 factors that represented $72.09 \%$ of total variance; whereas non-defeated animal's analysis was loaded with 5 factors that represented $85.46 \%$ of total variance. A 5 min non-aggressive interaction reduced the frequency of stretched attend behavior in the tunnel, whereas a single social defeat reduced time in the tunnel and increased time spent performing self-grooming in the home chamber without conditioning any other spatio-temporal and complementary measures. Together, these results suggest that different social interactions may modulate distinct behavioral profiles in animals when re-exposed to the context.
\end{abstract}

\section{Introduction}

Social interactions are present in several animal species (Alexander, 1974; Tinbergen, 1953) and can be classified as positive (e.g., interaction with a known individual, reproductive behavior of female rats receptive to males, parental care of females with their puppies) or negative interactions (e.g., interaction with an aggressive conspecific) (Kikusui et al., 2006; Armario et al., 1983a; Koolhaas et al., 1997). Many of these animal species, including human beings, live in a complex social environment in which individuals differ in their social interactions with other group members (Martinez et al., 1998). Some individuals are less exposed to aggression, whereas others face high and persistent levels of aggression, representing an intense source of social stress (Martinez et al., 1998). These social episodes are very important in modulating numerous behavioral and physiological processes (Sapolsky, 2001). Animals subjected to social defeat show impairment in exploratory behavior in the open-field (Kudriavtseva et al., 1991), increased submissive behavior, inhibition of sexual and aggressive behaviors (Kudriavtseva et al., 1996; Van de Poll et al., 1982; Yoshimura and Kimura, 1991), and increased anxiety assessed in the elevated plus maze (Costa et al., 2016; Rodgers and Cole, 1993), a widely used animal test of anxiety (e.g., Carobrez and Bertoglio, 2005). For this reason, conspecific confrontation has been used as a biologically relevant model of social stress in laboratory conditions (Martinez et al., 1998).

In humans, social stress is related with pathologies such as drug dependency, anxiety disorders and depression (Björkqvist, 2001; Chaouloff, 2013; Ehlers et al., 2000; Fuchs and Flugge, (2002); Goeders, 2002; Kessler, 1997; Koob and Thatcher-Britton, 1985; Monroe \& Simons, 1991; Paykel, 1978; Piazzaa and Le Moala et al., 1998; Sinha, 2008; Tidey and Miczek, 1997). Experimental models that validate these interactions are important tools to understand the neurobiology of several mental disorders. From a behavioral standpoint, it is well known that animals display various defensive reactions in response to threats, such as exposure to a predator or an aggressive conspecific (Edmunds, 1974). In rodents (e.g., rats and mice), these defense reactions classically include behaviors such as freezing, flight, defensive attacks and even death simulation (tonic immobility) (Blanchard et al., 1990). The behavioral choice exhibited by animals generally depends on their distance to the threatening stimulus and whether there is a

\footnotetext{
* Corresponding authors at: Laboratory of Pharmacology, School of Pharmaceutical Sciences, Universidade Estadual Paulista, Araraquara, SP 14801-902, Brazil.

E-mail addresses: arielamcrestani@gmail.com (A.M. Crestani), anac_cipri@yahoo.com.br (A.C. Cipriano), souzarn@fcfar.unesp.br (R.L. Nunes-de-Souza).
} 
route of escape (Blanchard et al., 1990). In this context, social stress, in the form of experience of defeat, is a potent situation for activating defense mechanisms (Rodgers and Randall, 1986) and also seems to be a suitable stressful stimulus to induce anxiogenic states in animals (Costa et al., 2016; Rodgers and Cole, 1993). Therefore, we hypothesized that different social interactions would elicit distinct behavioral profiles in animals and that the social defeat experience would provoke aversive contextual conditioning in the defeated mouse. To test these hypotheses, we investigated the behavioral profile of mice exposed to two types of social interaction (aggressive and non-aggressive) and whether a single exposure to the social defeat stress would be able to generate contextual avoidance. We employed factor analysis to carefully analyze the behavioral profile of socially defeated and non-defeated mice when re-exposed to the empty apparatus where the interaction occurred. To evaluate whether a single social defeat is able to induce contextual avoidance conditioning in mice, we also compared the behavioral profile displayed by non-defeated and socially defeated mice during re-exposure to the context where the social interaction was carried out (intergroup analysis). Finally, we compared the behavioral profile exhibited by mice during the habituation and re-exposure to the apparatus independently for each experimental group (DG and NDG, intragroup analysis).

\section{Material and methods}

\subsection{Subjects}

Subjects were male adult Swiss mice (from the animal facility at UNESP, Araraquara, SP, Brazil) weighing $30-35 \mathrm{~g}$ at testing. They were housed in groups of 10 per cage $(41 \times 34 \times 16 \mathrm{~cm})$ and maintained under a normal 12-h light cycle (lights on at 7:00 a.m.) in a temperature-controlled environment $\left(23 \pm 1{ }^{\circ} \mathrm{C}\right)$. Food and water were freely available except during the brief test period. All mice were experimentally naïve, and experimental sessions were carried out during the light phase of the cycle (08:00 a.m. to 02:00 p.m.).

\subsection{Choice of the aggressor conspecific}

Once the mice arrived at the animal house of the Laboratory of Pharmacology (School of Pharmaceutical Sciences, UNESP), they were housed in groups of 10 animals per cage $(45 \times 28 \times 13 \mathrm{~cm})$. In the period of habituation to local conditions, a trained observer identified the dominant mouse of each cage (defined as the individual that displayed spontaneous aggressive behavior against other animals in the home cage). These animals were then isolated in individual cages $(28 \times 17 \times 12 \mathrm{~cm})$ for at least 4 weeks, to intensify their aggressive behavior (Valzelli, 1985).

\subsection{Apparatus}

The apparatus was modified from the original "rat exposure test" developed and behaviorally validated by Yang et al. (2004). The apparatus has three interconnected chambers: home chamber (HC), tunnel (T), and the surface area (S), in order to measure the risk assessment behavior and spatio-temporal measures (Fig. 1). The surface area $(23 \times 24 \times 21 \mathrm{~cm})$ is a clear carbonate cage covered with a black polycarbonate lid. The home chamber $(7 \times 7 \times 12 \mathrm{~cm})$ is a black Plexiglas box covered with a lid. To facilitate videotaping, one side of the $\mathrm{HC}$ is made of clear Plexiglas. The HC is connected to the surface area by a clear Plexiglas tube tunnel $(4.4 \mathrm{~cm}$ in diameter, $13 \mathrm{~cm}$ in length and raised $1.5 \mathrm{~cm}$ above the floor of the two chambers).

\subsection{General procedure}

All tests were conducted under the illumination of a $100 \mathrm{~W}$ light lamp placed on room ceiling ( $24 \mathrm{~lx}$ on the floor of the apparatus). The apparatus was cleaned with $20 \%$ alcohol and dried with paper towels between trials. One horizontally mounted camera linked to a video monitor and DVD was used to record the experiment. Mouse behavior during the test was scored by an observer blinded to the experimental conditions using the software "X-plo-rat 2005", developed by Dr. Morato's group at Faculdade de Filosofia, Ciências e Letras de Ribeirão Preto, USP (the software can be freely downloaded at http://www. oocities.org/xplorat/). The behavioral parameters comprised both spatio-temporal and complementary measures. Spatio-temporal measures were the frequency of entries and time spent in each area of the apparatus (HC, T, and S), and latency (in seconds) to reach the S; whereas complementary measures comprised the frequency of stretched attend posture (SAP; exploratory posture in which the body is stretched forward but the animal's hind paws remain in position, followed by retracting to original position) in the HC, T and S, frequency of rearing (standing on rear limbs) in the $\mathrm{S}$, duration (in seconds) of self-grooming behavior [self-caring behavior directed to the body surface, generally following a cephalo-caudal direction: paw licking - nose and face wash - head wash - body wash - fur licking - leg licking - tail/ genitals licking and wash (Edmunds, 1974)] in the HC, T and S. The testing procedure consisted of five consecutive experimental days, divided into the following phases:

\subsubsection{Phase 1: habituation}

The habituation procedure occurred during the first three days of the experimental protocol with a $24 \mathrm{~h}$ interval between each habituation session. During habituation, each mouse was placed in the home chamber and was allowed to explore the empty apparatus for $10 \mathrm{~min}$. After analysis of data collected in habituation 3, animals were evenly distributed between the experimental groups (defeated and not defeated) taking as base the latency in seconds to reach the surface area in order to establish a homogeneous distribution of animals between both groups.

\subsubsection{Phase 2: social interaction test}

On the fourth day, each mouse was placed in the home chamber to freely explore the apparatus. Once the animal reached the surface area, the surface entrance was immediately closed and, then, an aggressive conspecific was introduced in the surface area and the agonistic encounter was started (defeated group - DG). The aggressive attacks were interrupted when the test animal displayed submissive posture [i.e. body lift on its hind legs, front paws outstretched toward the aggressor, retracted head and arched ears (Miczek, 1983)] for at least $5 \mathrm{~s}$ or after five minutes of interaction (which occurred first). Another group of animals was subjected to a similar procedure, except that a non-aggressive Swiss mouse was placed into the surface area for a 5-min period of social interaction (Non-defeated group; NDG).

\subsubsection{Phase 3: re-exposure test}

On the fifth day, mice were individually placed in the home chamber and could freely explore the entire apparatus for $10 \mathrm{~min}$. This phase was delineated to assess contextual avoidance conditioning.

\subsection{Data analysis}

Ninety non-defeated and eighty-nine defeated mice were habituated to the apparatus during the first three days of the protocol (Phase 1). On the fourth day (Phase 2), the animals were confined to the surface area where they experienced a social defeat session with an aggressive conspecific (animals from DG) or a non-aggressive interaction with a known conspecific (animals from NDG) as described in Section 2.4. To investigate any interrelation among the behaviors exhibited by defeated and non-defeated mice during the Phase 3, we first analyzed the data extracted from the 89 socially defeated and 90 non-defeated mice, doing three successive factor analyses for each experimental group. Initially, separate analyses were performed on the spatio-temporal 


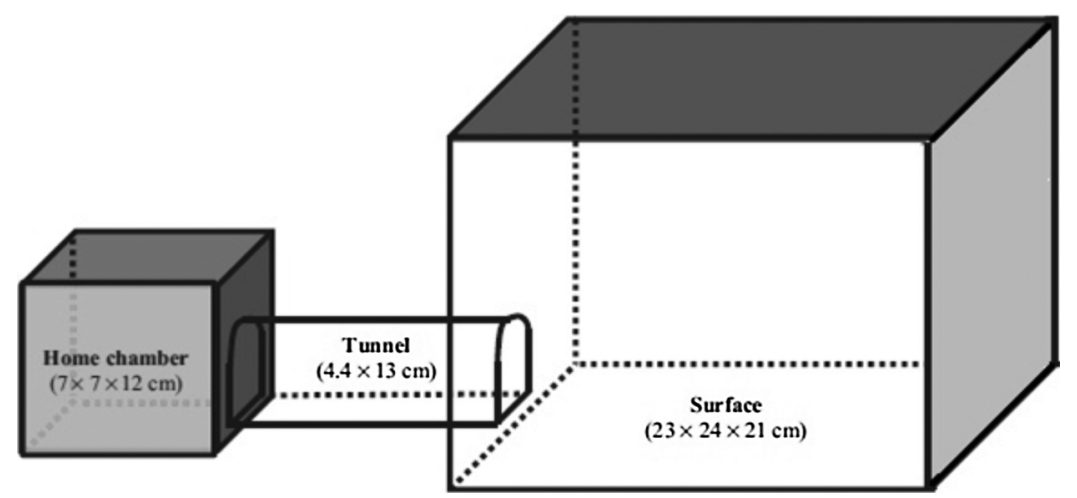

Fig. 1. A schematic view of the experimental apparatus composed of a home chamber, tunnel and a surface area (Adapted from Campos et al., 2013).

measures and on the complementary measures, aiming to clarify the behavioral structure and to reduce redundancy in the total of number of variables scored. The outcomes of these two analyses were then combined in a third factor analysis.

Factor analysis has been extensively used in several studies to characterize behavioral patterns of mice and rats in many paradigms such as the elevated plus maze, open elevated plus maze and rat exposure test (Campos et al., 2013; Sorregotti et al., 2013; Rodgers and Johnson, 1995; Lister, 1987). Through this analysis, it is possible to reduce a great number of observed variables to few factors. These factors summarize and explain a set of observed variables within a particular experimental group (Figueiredo-Filho and Silva-Júnior, 2010).

In Analysis 1, the factor analysis was performed by a principal component solution with orthogonal rotation (varimax) of the factor matrix. This method ensures that the extracted factors are independent and should, therefore, reflect distinct biological phenomena. The Kaiser test (eigenvalues $\geq 1$ ) was used to confirm the factors extracted. The factor loading of each behavioral item indicates how well that item correlates with that factor. Loading of \pm 1 indicates a perfect (positive/ negative) link, whereas a loading of less than 0.7 would suggest that the item is rather weakly linked to the factor. In this study, only loadings greater than 0.7 were used (Campos et al., 2013; Sorregotti et al., 2013).

Analysis 2 aimed to investigate whether the social interaction (5min non-aggressive interaction or a single social defeat) alters the behavioral profile presented in the apparatus, we performed a $2 \times 2$ (defeated vs. non-defeated, habituation 3 vs. re-exposure test) MANOVA followed by the Fisher's post hoc test comparing behavioral parameters shown by NDG $(\mathrm{N}=90)$ and $\mathrm{DG}(\mathrm{N}=89)$. For the "time factor" (habituation 3 vs. re-exposure test - intra-group analysis) a repeated measure analysis was carried out, considering that data from habituation 3 and re-exposure were obtained through the same animals for each group. In cases were significant effects were observed, the data were submitted to an ANCOVA analysis (main effect: treatment - aggressive or non-aggressive interaction, covariable: habituation 3) to identify if the behavioral profile exhibited on Habituation 3 may have influenced the difference between groups in re-exposure.

Initially, data were submitted to Levene's test to assess the equality of variances for both groups, which indicated a homogeneity of variance in group samples. A P $\leq 0.05$ was required for significance.

\section{Results}

\subsection{Analysis 1: behavioral profile analysis}

The results of Analysis 1 are summarized in the Tables 1-3. Factor analysis 1 (spatio-temporal measures) and 2 (complementary measures) from NDG were then combined in a third analysis (Table 1). The spatiotemporal and complementary analysis showed 5 factors that together
Table 1

Orthogonal factor loadings for spatio-temporal and complementary measures in non-defeated mice.

\begin{tabular}{|c|c|c|c|c|c|}
\hline \multirow[t]{2}{*}{ Behavioral parameter } & \multicolumn{5}{|l|}{ Factors } \\
\hline & 1 & 2 & 3 & 4 & 5 \\
\hline $\begin{array}{l}\text { Time spent in the home chamber } \\
\text { (s) }\end{array}$ & -0.908 & & & & \\
\hline Time spent in the tunnel (s) & & -0.893 & & & \\
\hline Time spent in the surface area (s) & +0.930 & & & & \\
\hline Total entries & & & -0.835 & & \\
\hline SAP total & & & & & +0.968 \\
\hline $\begin{array}{l}\text { Self-grooming in the home } \\
\text { chamber (s) }\end{array}$ & -0.769 & & & & \\
\hline Self-grooming in the tunnel (s) & & -0.942 & & & \\
\hline $\begin{array}{l}\text { Self-grooming in the surface area } \\
\text { (s) }\end{array}$ & +0.752 & & & & \\
\hline Rearing & & & -0.848 & & \\
\hline Latency to reach the surface & & & & +0.941 & \\
\hline$\%$ variance & 32.52 & 15.12 & 13.40 & 11.05 & 7.16 \\
\hline
\end{tabular}

Factors loadings greater than 0.7 are shown. Minus and plus signs indicate the direction of the particular loading. Criteria: eigenvalue $\geq 1$.

Table 2

Orthogonal factor loadings for spatio-temporal and complementary measures in socially defeated mice.

\begin{tabular}{|c|c|c|c|c|}
\hline \multirow[t]{2}{*}{ Behavioral parameter } & \multicolumn{4}{|l|}{ Factors } \\
\hline & 1 & 2 & 3 & 4 \\
\hline Time spent in the home chamber (s) & +0.965 & & & \\
\hline Time spent in the tunnel (s) & & -0.845 & & \\
\hline Time spent in the surface area (s) & -0.962 & & & \\
\hline Total entries & & -0.757 & & \\
\hline \multicolumn{5}{|l|}{ SAP in the home chamber } \\
\hline SAP in the tunnel & & & +0.735 & \\
\hline SAP in the surface area & & & +0.861 & \\
\hline Self-grooming in the home chamber (s) & +0.806 & & & \\
\hline \multicolumn{5}{|l|}{ Self-grooming in the tunnel (s) } \\
\hline \multicolumn{5}{|l|}{ Self-grooming in the surface area (s) } \\
\hline \multicolumn{5}{|l|}{ Rearing } \\
\hline Latency to reach the surface & & & & +0.879 \\
\hline$\%$ variance & 32.52 & 15.12 & 13.40 & 11.05 \\
\hline
\end{tabular}

Factors loadings greater than 0.7 are shown. Minus and plus signs indicate the direction of the particular loading. Criteria: eigenvalue $\geq 1$.

represent $85.46 \%$ of total variance. Factor 1 was loaded with time spent in the home chamber and in the surface area, and time spent performing self-grooming in the home chamber. Factor 2 was negatively loaded with time spent in the tunnel and self-grooming in the same compartment; whereas factor 3 was negatively loaded with total entries 
Table 3

Ethological description and comparison of behavior displayed by non-defeated $(\mathrm{n}=90)$ and socially defeated $(\mathrm{N}=89)$ mice on the re-exposure test.

\begin{tabular}{|c|c|c|c|}
\hline Parameter & Non-defeated mice Mean \pm SEM & Socially defeated mice Mean \pm SEM & $\mathrm{P}$ value \\
\hline \multicolumn{4}{|l|}{ Time spent (s) } \\
\hline Home chamber & $229.89 \pm 12.84$ & $275.03 \pm 14.17$ & $\mathrm{P}=0.045^{*}$ \\
\hline Tunnel & $50.45 \pm 3.38$ & $44.97 \pm 2.44$ & $P=0.36$ \\
\hline Protected areas (home chamber + tunnel) & $280.33 \pm 12.96$ & $320.0 \pm 13.29$ & $\mathrm{P}=0.03^{*}$ \\
\hline Surface area & $319.67 \pm 12.29$ & $279.99 \pm 13.30$ & $\mathrm{P}=0.06$ \\
\hline \multicolumn{4}{|l|}{ Frequency of entries } \\
\hline Home chamber & $13.39 \pm 0.63$ & $11.49 \pm 0.53$ & $P=0.21$ \\
\hline Tunnel & $26.08 \pm 1.26$ & $22.21 \pm 1.12$ & $\mathrm{P}=0.21$ \\
\hline Protected areas (home chamber + tunnel) & $39.47 \pm 1.87$ & $33.69 \pm 1.63$ & $P=0.21$ \\
\hline Surface area & $13.57 \pm 0.64$ & $11.66 \pm 0.61$ & $P=0.24$ \\
\hline \multicolumn{4}{|l|}{ Stretched Attend Posture (Freq.) } \\
\hline Home chamber & $0.13 \pm 0.04$ & $0.29 \pm 0.098$ & $P=0.41$ \\
\hline Tunnel & $0.81 \pm 0.15$ & $1.28 \pm 0.315$ & $P=0.27$ \\
\hline Protected areas (home chamber + tunnel) & $0.94 \pm 0.17$ & $1.57 \pm 0.342$ & $P=0.08$ \\
\hline Surface area & $0.89 \pm 0.17$ & $1.22 \pm 0.254$ & $P=0.73$ \\
\hline \multicolumn{4}{|l|}{ Self-Grooming (s) } \\
\hline Home chamber & $69.94 \pm 7.85$ & $112.94 \pm 10.93$ & $P=0.019$ \\
\hline Tunnel & $0.71 \pm 0.5$ & $0.22 \pm 0,197$ & $\mathrm{P}=0.87$ \\
\hline Protected areas (home chamber + tunnel) & $70.66 \pm$ & $113.16 \pm 10.94$ & $\mathrm{P}=0.018^{*}$ \\
\hline Surface area & $38.04 \pm 7.1$ & $23.91 \pm 4.83$ & $\mathrm{P}=0.42$ \\
\hline \multicolumn{4}{|l|}{ Rearing (Freq.) } \\
\hline Surface area & $43.12 \pm 2.3$ & $39.70 \pm 2.08$ & $P=0.29$ \\
\hline \multicolumn{4}{|l|}{ Latency to reach the surface (s) } \\
\hline Surface area & $7.83 \pm 3.42$ & $27.65 \pm 8.59$ & $\mathrm{P}=0.21$ \\
\hline
\end{tabular}

This dataset was used in factor analysis reported in Tables 1 and 2, and also in the MANOVA and ANCOVA analyses. Values represent mean \pm SEM. *P $<0.05$ compared to non-defeated group through MANOVA followed by the Fisher's post test.

and rearing behavior. Factors 4 and 5 were positively loaded with a single behavior measure each one; latency to reach the surface area and total SAP, respectively. On factor 1 , time spent in home chamber and self-grooming in the same compartment were positively loaded, whereas this factor was negatively loaded with time spent in the surface area and self-grooming also in the same area. There were no co-loadings for any measure.

Factor analyses for behavioral measures from DG are presented in Table 2. The results obtained from factor analysis 1 (spatio-temporal measures) and 2 (complementary measures) were then combined in a third-factor analysis (Table 2). This analysis resulted in four different factors that together represented $72.09 \%$ of total variance. Factor 1was positively loaded with time spent in the home chamber and selfgrooming in the same compartment; besides, this factor was also negatively loaded with time spent in the surface area. Factor 2 was negatively loaded with time spent in the tunnel and total entries, whereas factor 3 was positively loaded with SAP in the tunnel and in the surface. Latency to reach the surface positively loaded alone on factor 4 .

Table 3 shows the behavioral profile of NDG and DG on the reexposure test. Animals from the DG showed a slight preference for the protected areas (home chamber + tunnel; 53.3\%) where they mainly displayed self-grooming behavior $(\approx 35 \%)$, whereas non-defeated mice spent more time in the surface area rather than in protected areas.

\subsection{Analysis 2: contextual aversive conditioning in defeated and non- defeated mice}

At first, it was performed an analysis using a $2 \times 2$ (defeated vs. non-defeated, habituation 3 vs. re-exposure test) factorial analysis MANOVA followed by the Fisher's post hoc. From an intergroup perspective of analysis (comparing data re-exposure test between the two experimental groups), this statistical approach showed that animals from NDG and DG differed in the re-exposure test for only two behavioral measures: time spent in the home chamber $(p=0.04)$ and time spent performing self-grooming also in the home chamber $(p=0.02)$, whereas all other behaviors did not change after distinct social interactions (Table 3).

This analysis also revealed that experimental groups differed in some behaviors during habituation 3: time spent in the home chamber $\left(\mathrm{NDG}=220.8 \pm 13.03 \quad\right.$ and $\quad \mathrm{DG}=277.83 \pm 13.23 \quad ; \quad \mathrm{F}_{(1}$, $178)=10.511, \mathrm{p}=0.01)$, and in surface area (NDG $=323.9 \pm 12.7$ and $\left.\mathrm{DG}=268.79 \pm 13.02 ; \mathrm{F}_{(1,178)}=9.838, \mathrm{p}=0.01\right)$; and time spent performing self-grooming in the home chamber $\left(\mathrm{NDG}=77.13 \pm 9.3\right.$ and $\mathrm{DG}=117.81 \pm 10.78 ; \mathrm{F}_{(1,178)}=14.189$, $\mathrm{p}=0.01$ ). Other behavioral measures did not show between-group differences in habituation 3. These results indicate that the adopted criterion (i.e. the latency to reach the surface in habituation 3) to homogeneously distribute the animals into the experimental group was not effective. Considering these results, it was performed a covariance analysis-ANCOVA (main effect: treatment - aggressive or non-aggressive interaction, covariable: habituation 3). The purpose of this statistical approach was to evaluate whether the differences observed for time and self-grooming in the home chamber during re-exposure were due to treatment effect (aggressive or non-aggressive interaction) or to the difference already existing in habituation 3.

Thus, ANCOVA revealed significant effect for treatment (aggressive or non-aggressive interaction) in self-grooming behavior in the home chamber; $\mathrm{F}_{(2,178)}=4.47, \mathrm{p}<0.05$. Regarding the variable "time spent in the home chamber", ANCOVA revealed a lack of effect for treatment; $\mathrm{F}_{(2,178)}=0.64, \mathrm{p}>0.05$.

It was also possible to analyze these data from an intra-group perspective, comparing data from habituation 3 and re-exposure test independently for each experimental group and assessing whether the employed intervention (5- min non-aggressive interaction or a single social defeat) was able to change the behavioral profile of mice re-exposed to the context in which the interaction occurred.

The $2 \times 2$ MANOVA with Fisher's post hoc test revealed lack of effect when habituation 3 and re-exposure test for all spatio-temporal measures in NDG were compared: time spent in the home chamber, tunnel and surface area $(\mathrm{p}=0.38, \mathrm{p}=0.1$ and $\mathrm{p}=0.64$, respectively), frequency of entries in the home chamber, tunnel, and surface area ( $\mathrm{p}=0.08, \mathrm{p}=0.08$ and $\mathrm{p}=0.22$, respectively), and latency to reach 
A. Time in $\mathrm{HC}$

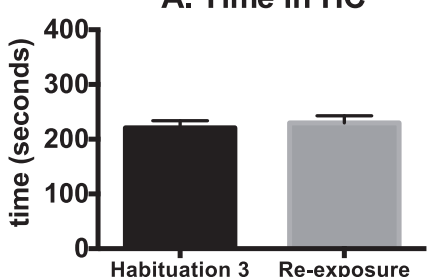

Habituation 3 Re-exposure
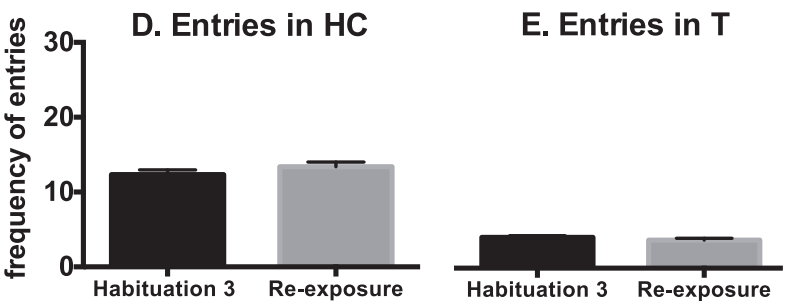

G. Latency

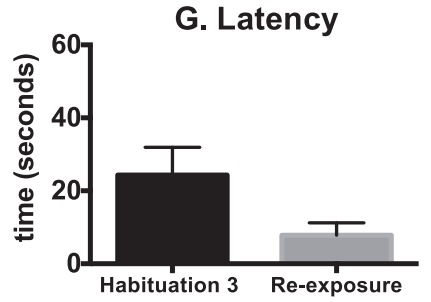

A. SAP in HC
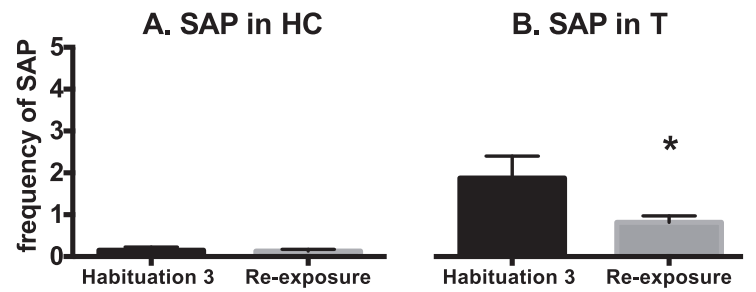

D. Self-grooming in $\mathrm{HC}$

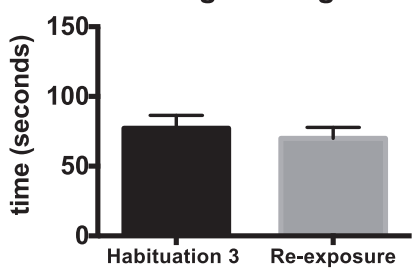

G. Rearing in $S$

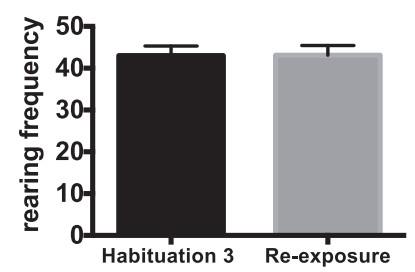

B. Time in $\mathrm{T}$

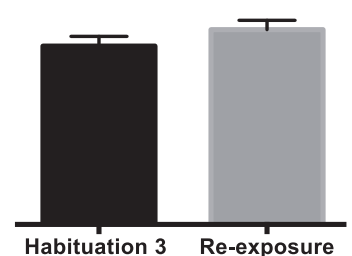

E. Entries in T

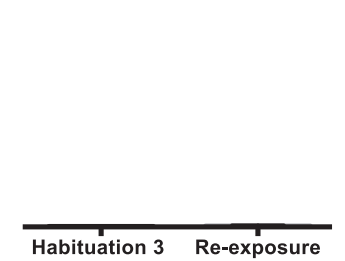

Habituation 3 Re-exposure

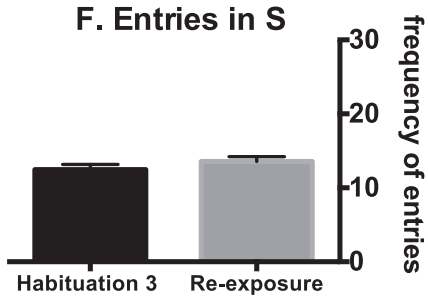

C. SAP in S
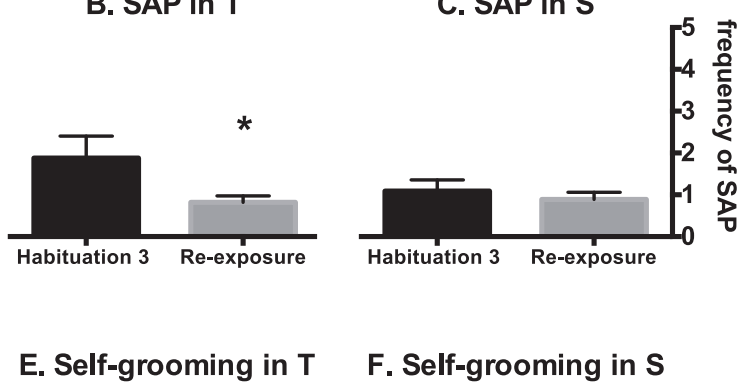

\section{F. Self-grooming in $\mathbf{S}$}

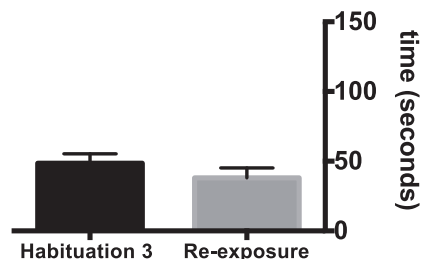

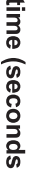

Fig. 2. Spatio-temporal behavioral measures exhibited by non-defeated group (NDG, $\mathrm{n}=91$ ) during habituation 3 and re-exposure test. Time in seconds spent in the home chamber (A), tunnel (B) surface area (C); frequency of entries in the home chamber (D), tunnel (E) and surface area (F); latency in seconds to reach the surface area (G). Bars represent mean \pm SEM. ${ }^{*} \mathrm{P}<0.05$.
Fig. 3. Complementary behavioral measures exhibited by non-defeated group (NDG, $\mathrm{n}=91$ ) during habituation 3 and re-exposure test. SAP frequency in the home chamber (A), tunnel (B) and surface area (C); time in seconds spent performing self-grooming in the home chamber (D), tunnel (E) and surface area (F); frequency of rearing behavior in the surface area (G). Bars represent mean \pm SEM. ${ }^{*} \mathrm{P}<0.05$. 
A. Time in $\mathrm{HC}$

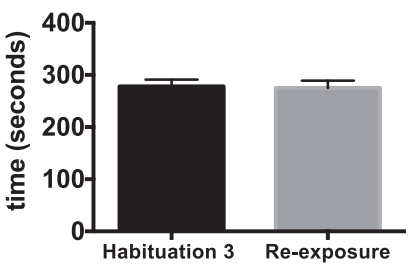

D. Entries in $\mathrm{HC}$
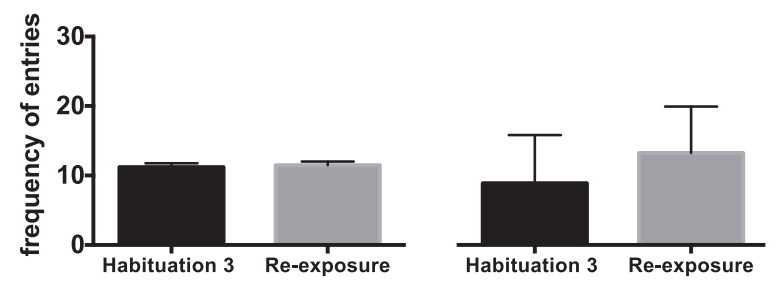

E. Entries in T

\section{G. Latency}

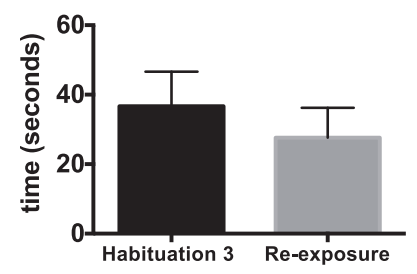

C. Time in S

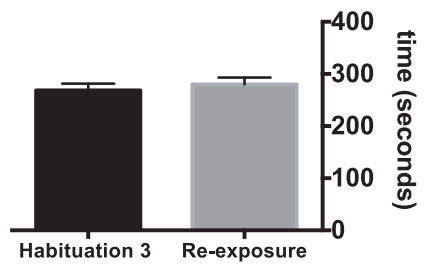

F. Entries in S

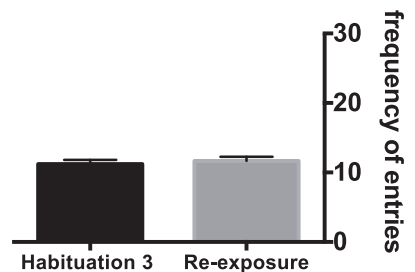

Fig. 4. Spatio-temporal behavioral measures exhibited by defeated group ( $D G, n=89)$ during habituation 3 and re-exposure test. Time in seconds spent in the home chamber (A), tunnel (B) surface area (C); frequency of entries in the home chamber (D), tunnel (E) and surface area (F); latency in seconds to reach the surface area (G). Bars represent mean \pm SEM. ${ }^{*} \mathrm{P}<0.05$. the surface area ( $p=0.09$ - Fig. 2). Additionally, a lack of effect was observed between habituation 3 and re-exposure test for almost all complementary measures: SAP in home chamber $(\mathrm{p}=0.7)$ and in the surface area $(p=0.52)$; self-grooming in the home chamber $(p=0.62)$, in tunnel $(\mathrm{p}=0.17)$, and surface area $(\mathrm{p}=0.09)$; and rearing behavior ( $p=0.08$ - Fig. 3 ). The only complementary measure that showed an effect for NDG was the SAP in the tunnel, that had a lower frequency in the re-exposure test when compared to the habituation $3(\mathrm{p}=0.03-$ Fig. 3).

For the DG, when behaviors exhibited in habituation 3 and re-exposure test were compared, the analysis showed a lack of effect for almost all spatio-temporal behaviors: time spent in the home chamber and in surface area $(\mathrm{p}=0.87$ and $\mathrm{p}=0.42$, respectively), frequency of entries in home chamber $(\mathrm{p}=0.42)$, tunnel $(\mathrm{p}=0.34)$ and surface area $(p=0.29)$, and latency to reach the surface area $(p=0.24)$ (Fig. 4). The only spatio-temporal behavior changed by the social defeat was the time spent in the tunnel, that decreased $(\mathrm{p}=0.02)$. For complementary measures, no effect was observed: SAP frequency in home chamber $(\mathrm{p}=0.14)$, in tunnel $(\mathrm{p}=0.053)$, and in surface area $(\mathrm{p}=0.7)$; selfgrooming in the home chamber ( $\mathrm{p}=0.68)$, in tunnel $(\mathrm{p}=0.88)$, and in surface area $(p=0.33)$; and rearing behavior $(p=0.07$ - Fig. 5$)$.

\section{Discussion}

The main aim of this study was to evaluate the effects of different social interactions (i.e. aggressive and non-aggressive) on the behavioral profile of animals re-exposed to the context in which the interaction occurred.

Through factor analysis was possible to observe differences in the behavioral profile of defeated and non-defeated animals when re-exposed to the context of the social interaction. It was also noticed that socially defeated animals spent more time performing self-grooming in the home chamber than non-defeated animals during re-exposure test.
Also, socially defeated animals spent less time in the tunnel in the reexposure test compared to habituation 3. A reduction in frequency of SAP in the tunnel was also observed in NDG when comparing behaviors presented in the habituation 3 and re-exposure test.

Comparing the final factor analyses (spatio-temporal and complementary measures) for each group, it is possible to affirm that NDG had a factor analysis with 5 factors that together represent $85.46 \%$ of all behavioral measures displayed by animals (see Table 1). Regarding the results displayed by mice of the DG, factor analysis showed 4 factors that together represent $72.09 \%$ of their behavior repertory. The lower number of factors and \% variance in DG compared to those raised in NDG can be attributed to the fact that some behavioral measures were not sufficiently relevant to any of the biological factors resulted from DG factor analysis, such as SAP in the home chamber, self-grooming behavior in the tunnel and surface area, and rearing.

Factor 1 on the final analyses of both experimental groups seems to reflect animal's permanency in the main compartments of the apparatus, because time spent in the home chamber and in surface area were strongly but negatively loaded on both analyses. In addition to these two behavioral measures, factor 1 in NDG also loaded with selfgrooming behavior in the home chamber and surface area; whereas in DG factor 1 was additionally loaded with self-grooming in the home chamber only. This distinct outcome on factor 1 observed in NDG and DG is suggestive that while DG animals recognized home chamber as a safe area to exhibit a recuperative behavior such as self-grooming (Domjan, 2014; Spruijt et al., 1992), NDG did not have to search for safe places in the apparatus probably because they did not suffer an aggressive experience.

Factors 2 of both experimental groups, although showed similar participation in total variance (i.e. $15.12 \%$ ), had their behavioral measures loading slightly different. In NDG, factor 2 loaded with time spent in the tunnel and self-grooming behavior in the same area, that were strongly and positively correlated; indicating that this factor may 
A. SAP in HC

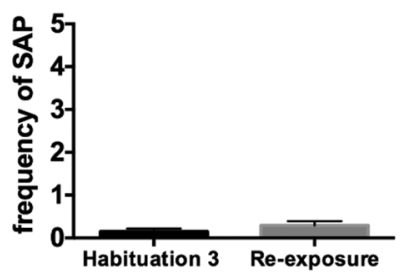

D. Self-grooming in HC

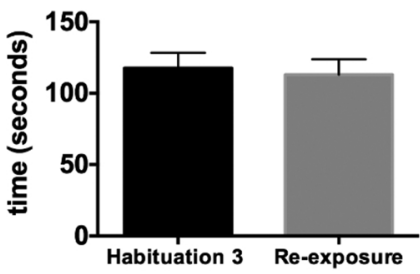

G. Rearing in $\mathbf{S}$

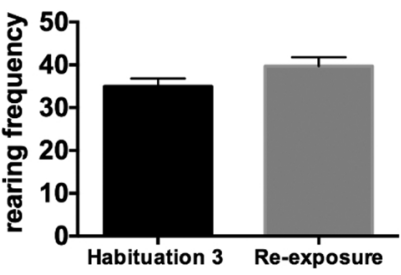

B. SAP in T

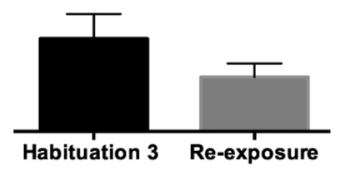

E. Self-grooming in T
C. SAP in S

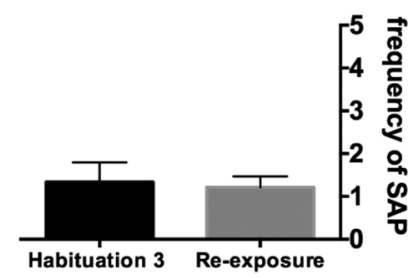

F. Self-grooming in $\mathbf{S}$
Fig. 5. Complementary behavioral measures exhibited by defeated group (DG, $\mathrm{n}=89$ ) during habituation 3 and re-exposure test. SAP frequency in the home chamber (A), tunnel (B) and surface area (C); time in seconds spent performing self-grooming in the home chamber (D), tunnel (E) and surface area (F); frequency of rearing behavior in the surface area (G). Bars represent mean \pm SEM.

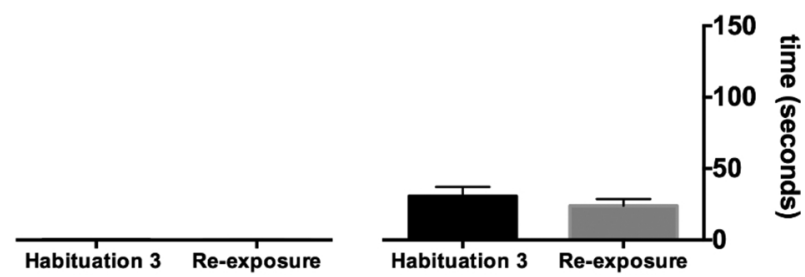

be reflecting time and behaviors (i.e. self-grooming) presented in a passage/transition environment. Whereas for DG, factor 2 was loaded with time spent in the home chamber and total entries. Considering that the frequency of entries is generally used as a locomotion measure (Campos et al., 2013), this factor may represent locomotion/exploratory behaviors. In NDG, this exploratory/locomotor profile was observed in factor 3 that was also loaded with total entries and rearing behavior. In contrast, Factor 3 from DG possibly resumes an emotional factor because it was loaded with SAP behavior in the tunnel and in the surface compartments. The NDG also had a factor with emotional substrate; factor 5 was loaded with a single measure: total SAP. Despite some similarities in the SAP exhibition observed in DG and NDG interactions, factor 3 from DG loaded with SAP in the tunnel and in the surface area, indicating that these risk assessment measures were strongly correlated only when the animals had experienced an aggressive interaction. In relation to factor 4 , it was loaded with latency to reach the surface area for both NDG and DG.

Lastly, through the comparison between final factor analyses of NDG and DG is possible to see that different social interactions (i.e. aggressive and non-aggressive) modulate behavioral profile presented by the animals when re-exposed to the context where they experienced social interactions, not just by the number of factors extracted from the behavioral measure, but also by the content of each factor.

The second analysis aimed to determine whether a single social defeat could produce contextual avoidance in socially defeated mice. A $2 \times 2$ (defeated vs. non-defeated, habituation 3 vs. re-exposure test) MANOVA with Fisher's post hoc, followed by an ANCOVA analysis revealed that socially defeated animals spent more time performing self-grooming in the home chamber during the re-exposure test than non-defeated animals, and less time in the tunnel in the re-exposure test compared to the habituation 3 (intra-group analysis). Albeit the MANOVA analysis had indicated that time spent in the home chamber during re-exposure test was higher for DG compared to the NDG; this effect could not be considered, because ANCOVA analysis indicated that this result was influenced by a pre-existent difference between these groups (NDG and DG) in habituation 3.

Although self-grooming has the essential function of caring for and protecting the body surface, its occurrence is affected by emotional factors (Estanislau et al., 2013). It is known that the neural substrates that underlie behaviors directed to the body surface overlaps other substrates involved in the processing of stressful stimuli (Spruijt et al., 1992). It has been long known that self-grooming behavior can occur in various contexts and generally is increased in two opposite situations in which high and low levels of stress are present (Kalueff and Tuohimaa, 2004). Our results are consistent with previous findings that have demonstrated that socially defeated rats showed an increase in selfgrooming behavior 25-30 min after the aggressive interaction when compared to non-defeated rats (Van Erp et al., 1994).

Apparently, the expression of self-grooming behavior in rodents is more related to the period after the peak of exposure to the stressful source rather than with the period of high levels of fear/anxiety. Therefore, it is likely that self-grooming expression reflects the process of arousal reduction due to the termination of exposure to stressful source or habituation to an adverse situation (Spruijt et al., 1992). Considering that in the present study, socially defeated mice increased self-grooming in the home chamber (protected area) during the re-exposure to the same context (but without the presence of the aggressive conspecific), it seems reasonable to suggest that this behavioral parameter may be related to the arousal reduction.

Though socially defeated animals spent more time performing selfgrooming in the home chamber than non-defeated animals, a single social defeat failed to induce contextual conditioning for classical spatio-temporal and complementary measures such as preference for the home chamber, avoidance for the surface area and increased SAP behavior. Faced with this mild effect revealed by MANOVA/ANCOVA intergroup analysis, data were then explored under a within-group 
(intragroup) approach, in order to verify if there were differences between habituation 3 and re-exposure test for a single experimental group (i.e. DG or NDG). Doing so, we were able to observe that animals from NDG reduced SAP frequency in the tunnel after a 5-min interaction with a known conspecific. It is likely that this reduction is related to a rewarding valence of the non-aggressive interaction between familiar conspecifics. In brief, it is known that social animals communicate each other in order to cooperate and manage the group in such way that is beneficial to either group member. These social interactions are essential not only for cooperation between animals, but also for protection of possible treats in the environment (Kikusui et al., 2006). Behavioral approaches also show that when conspecifics animals are together in a semi-natural environment, there is a reduction in their corticosterone and stress levels when compared to isolated animals (Armario et al., 1983a,b; Kikusui et al., 2006). Furthermore, this effect seems to persist even $24 \mathrm{~h}$ after the interaction, because animals reduced SAP in the tunnel when re-exposed to the interaction context.

Regarding DG, although the animals were socially defeated exclusively in the surface area, they did not show place avoidance for the surface area, suggesting that a single social defeat failed to induce conditioned place aversion (for surface area). A similar result was shown by Faturi et al. (2014), who demonstrated that single socially defeated rats did not exhibit place avoidance when re-exposed to the apparatus containing fresh bedding (i.e. without the aggressor's odor). On the other hand, animals re-exposed to the apparatus with soiled bedding (i.e. with the aggressor's odor) showed avoidance for the compartment where they have been attacked. In other words, they preferred to stay in the home chamber. Taken together, these results are suggestive that the presence of additional contextual cues (e.g., aggressor's scent) may be necessary to elicit contextual avoidance conditioning, particularly when a single social defeat is employed.

Importantly, the total entries in the three different compartments of the apparatus as well as the frequency of rearing expressed by DG did not change between habituation 3 and re-exposure test, suggesting that a single social defeat is not able to alter or impair general locomotion and exploratory (total and vertical) activity. These results corroborate those reported by Faturi et al. (2014), who did not find any differences in the frequency of rearing and entries in the apparatus compartments exhibited by defeated and non-defeated rats during re-exposure to the context. Regarding SAP, we did not observe any difference in its frequency, in any region (home chamber, tunnel or surface area) between habituation 3 and re-exposure test for DG. A similar result was shown by Faturi et al. (2014) using rats as experimental subjects.

The present study also raised several critical points that should be taken into account by researchers who intend to investigate contextual avoidance acquisition using the social defeat paradigm. First, mice exhibit individual degrees (levels) of defensiveness when confronted to an aggressive conspecific. For instance, whereas some animals need highly intense aggressive interaction to exhibit submissive posture, other mice reach this criteria during the first contacts with an aggressive conspecific. These behavioral discrepancies lead to different strategies for coping with social stress and may interfere with the behavioral profile of these animals when re-exposed to the apparatus. Second, the use of a single acute aggressive interaction may not be enough to ensure a strong contextual avoidance conditioning. Mice are social animals that live in colonies with well-established social hierarchies and it is known that aggressive interactions with conspecific are very frequent in a group living together. Thus, it is possible that a single aggressive interaction is not stressful enough to elicit contextual conditioned responses. Alternative procedures to strengthen the contextual conditioning in socially defeated mice might be the use of additional environmental cues during testing and retesting sessions in the apparatus, as reported by Faturi et al. (2014), and/or even increasing the social stress intensity.

\section{Conclusions}

In conclusion, using the statistical resource of factor analysis, our work traced behavioral profiles of socially defeated and non-defeated mice in a paradigm involving social defeat, and evidenced that exhibited behavioral profiles are different between groups that were subjected to distinct social interactions. We also showed that socially defeated animals spent more time performing self-grooming in the home chamber (protected area) than non-defeated animals during reexposure test. This result may indicate that defeated animals recognize the $\mathrm{HC}$ as a protected region to exhibit recovery behavior, such as selfgrooming. A 5-min non-aggressive interaction between familiar conspecifics reduced SAP in the tunnel when animals were re-exposed to the interaction context after $24 \mathrm{~h}$, suggesting a possible "social buffering" effect in these animals. Furthermore, a single social defeat reduced time spent in the tunnel, without compromising locomotor activity and exploratory behaviors. However, it failed to induce contextual avoidance conditioning for classic spatio-temporal and complementary parameters such as place avoidance (time spent in the compartments) and risk assessment measures such as SAP. Moreover, the use of a single and acute source of stress as well as the absence of any other cue in the re-exposure test may have been contributed to the response exhibited by defeated mice. Alternatives to render the social defeat protocol more robust to elicit contextual avoidance conditioning might be (a) grouping more responsive animals to social defeat; (b) increasing the number of social aggressive encounters and/or (c) adding further cues to facilitate contextual learning.

\section{Compliance with ethical standards}

All applicable international, national, and/or institutional guidelines for the care and use of animals were followed. All procedures performed in studies involving animals were in accordance with the ethical standards of the institution or practice at which the studies were conducted (approved by the Sao Paulo State University Research Ethics Committee - CEP/FCF/Car protocol 47/2013).

This article does not contain any studies with human participants performed by any of the authors.

\section{Funding}

This work was supported by São Paulo Research Foundation (FAPESP, 2013/01383-6), National Council for Scientific and Technological Development (CNPq, 478696/2013-2) and PADC/FCFUNESP. Ariela M. Crestani and Ana C. Cipriano received scholarship from CNPq (131661/2013-2) and FAPESP (2011/04561-1), respectively, and Ricardo L. Nunes-de-Souza was supported by a CNPq fellowship (306556/2015-4).

\section{References}

Alexander, R.D., 1974. The evolution of social behavior. Ann. Rev. Ecol. Syst. 5, 325-383. Armario, A., Luna, G., Balasch, J., 1983a. The effect of conspecifics on corticoadrenal response of rats to a novel environment. Behav. Neural Biol. 37, 332-337. http://dx. doi.org/10.1016/S0163-1047(83)91425-5.

Armario, A., Ortiz, R., Balasch, J., 1983b. Corticoadrenal and behavioral-response to open-field in pairs of male rats either familiar or non-familiar to each other. Experientia 39, 1316-1317. http://dx.doi.org/10.1007/BF01990391.

Björkqvist, K., 2001. Social defeat as a stressor in humans. Physiol. Behav. 7, 435-442. http://dx.doi.org/10.1016/S0031-9384(01)00490-5.

Blanchard, R.J., Blanchard, D.C., Rodgers, R.J., Weiss, S.M., 1990. The characterization and modelling of antipredator defensive behavior. Neurosci. Biobehav. Rev. 14, 491-496. http://dx.doi.org/10.1016/S0149-7634(05)80069-7.

Campos, K.F.C., Amaral, V.C.S., Rico, J.L., Miguel, T.T., Nunes-de-Souza, R.L., 2013 Ethopharmacological evaluation of the rat exposure test: a prey-predador interaction test. Behav. Brain Res. 240, 160-170. http://dx.doi.org/10.1016/j.bbr.2012.11.023.

Carobrez, A.P., Bertoglio, L.J., 2005. Ethological and temporal analyses of anxiety-like behavior: the elevated plus-maze model 20 years on. Neurosci. Biobehav. Rev. 29, 1193-1205. http://dx.doi.org/10.1016/j.neubiorev.2005.04.017.

Chaouloff, F., 2013. Social stress models in depression research: what do they tell us? Cell 
Tissue Res. 354, 179-190. http://dx.doi.org/10.1007/s00441-013-1606-x.

Costa, N.S., Vicente, M.A., Cipriano, A.C., Miguel, T.T., Nunes-de-Souza, R.L., 2016. Functional lateralization of the medial prefrontal cortex in the modulation of anxiety in mice: left or right? Neuropharmacology 108, 82-90. http://dx.doi.org/10.1016/j. neuropharm.2016.04.011.

Domjan, M.P., 2014. The Principles of Learning and Behavior, 7th ed. Wadsworth, Stanford.

Edmunds, M., 1974. Defence in Animals: A Survey of Anti-Predator Defences. Longman, Burnt Mill. (England).

Ehlers, A., Maercker, A., Boos, A., 2000. Posttraumatic stress disorder following political imprisonment: the role of mental defeat, alienation, and perceived permanent change. J. Abnorm. Psychol. 109, 45-55. http://dx.doi.org/10.1037/0021-843X.109. 1.45 .

Estanislau, C., Díaz-Morán, S., Cañete, T., Blázquez, G., Tobeña, A., Fernández-Teruel, A., 2013. Context-dependent differences in grooming behaviour among the NIH heterogeneous stock and the Roman high- and low- avoidance rats. Neurosci. Res. 77, 187-201. http://dx.doi.org/10.1016/j.neures.2013.09.012.

Faturi, C.B., Rangel, M.J., Baldo, M.V.C., Canteras, N.S., 2014. Functional mapping of the circuits involved in the expression of contextual fear responses in socially defeated animals. Brain Struct. Funct. 219, 931-946. http://dx.doi.org/10.1007/s00429-0130544-4.

Figueiredo Filho, D.B., Silva Júnior, J. Ada, 2010. Visão além do alcance: uma introdução à análise fatorial. Opinião Pública 16, 160-185. http://dx.doi.org/10.1590/S010462762010000100007.

Fuchs, E., Flugge, G., 2002. Social stress in tree shrews: effects on physiology, brain function, and behavior of subordinate individuals. Pharmacol. Biochem. Behav. 73, 247-258. http://dx.doi.org/10.1016/S0091-3057(02)00795-5.

Goeders, N.E., 2002. Stress and cocaine addiction. J. Pharmacol. Exp. Ther. 301, 785-789. http://dx.doi.org/10.1124/jpet.301.3.785.

Kalueff, A.V., Tuohimaa, P., 2004. Grooming analysis algorithm for neurobehavioural stress research. Brain Res. Protoc. 13, 151-158. http://dx.doi.org/10.1016/j. brainresprot.2004.04.002.

Kessler, R.C., 1997. The effects of stressful life events on depression. Annu. Rev. Psychol. 48, 191-214. http://dx.doi.org/10.1146/annurev.psych.48.1.191.

Kikusui, T., Winslow, J.T., Mori, Y., 2006. Social buffering: relief from stress and anxiety. Phil. Trans. R. Soc. 361, 2215-2228. http://dx.doi.org/10.1098/rstb.2006.1941.

Koob, G.F., Thatcher-Britton, K., 1985. Stimulant and anxiogenic effects of corticotropin releasing factor. Prog. Clin. Biol. Res. 192, 499-506.

Koolhaas, J.M., de Boer, S.F., de Ruiter, A.J.H., Meerlo, P., Sgoifo, A., 1997. Social stress in rats and mice. Acta Physiol. Scand. 161, 69-72.

Kudriavtseva, N.N., Amstislavskaia, T.G., Avgustinovich, D.F., Bakshtanovskaia, I.V., Lipina, T.V., Gorbach, O.V., Koriakina, L.A., 1996. The effect of the repeated experience of victories and defeats in social conflicts on the function of the brain serotoninergic system in male mice. Zhurnal Visshey Nervnoy Deyatelnosti Imeni I P Pavlova 46, 1088-1096.

Kudriavtseva, N.N., Bakshtanovskaya, I.V., Koryakina, L.A., 1991. Social model of depression in mice of C57BL/6J strain. Pharmacol. Biochem. Behav. 38, 315-320. http://dx.doi.org/10.1016/0091-3057(91)90284-9.

Lister, R.G., 1987. The use of a plus-maze to measure anxiety in the mouse. Psychopharmacology 92, 180-185.

Martinez, M., Phillips, P.J., Herbert, J., 1998. Adaptation in patterns of c-fos expression in the brain associated with exposure to either single or repeated social stress in male rats. Eur. J. Neurosci. 10, 20-33. http://dx.doi.org/10.1046/j.1460-9568.1998. 00011.x.

Monroe, S., Simons, A., 1991. Diathesis-stress theories in the context of life stress research: implications for depressive disorders. Psychol. Bull. 110, 406-425.

Miczek, K.A., 1983. Ethological analysis of drug action on aggression and defense. Prog Neuro-Psychopharmacol. Biol. Psychiatry 7, 519-524. http://dx.doi.org/10.1016/ 0278-5846(83)90019-2.

Paykel, E.S., 1978. Contribution of life events to causation of psychiatric illness. Psychol. Med. 8, 245-253. http://dx.doi.org/10.1017/S003329170001429X.

Piazzaa, P.V., Le Moala, M., 1998. The role of stress in drug self-administration. Trends Pharmacol. Sci. 19, 67-74. http://dx.doi.org/10.1016/S0165-6147(97)01115-2.

Rodgers, R.J., Cole, J.C., 1993. Anxiety enhancement in the murine elevated plus maze by immediate prior exposure to social stressors. Physiol. Behav. 53, 383-388. http://dx. doi.org/10.1016/0031-9384(93)90222-2.

Rodgers, R.J., Johnson, N.J.T., 1995. Factor analysis of spatiotemporal and ethological measures in the murine plus-maze test of anxiety. Pharmacol. Biochem. Behav. 52, 297-303. http://dx.doi.org/10.1016/0091-3057(95)00138-M.

Rodgers, R.J., Randall, J.I., 1986. Acute non-opioid analgesia in defeated male mice. Physiol. Behav. 36, 947-950. http://dx.doi.org/10.1016/0031-9384(86)90458-0.

Sapolsky, R.M., 2001. Physiological and pathophysiological implications of social stress in mammals. In: McEwen, B.S., Goodman, H.M. (Eds.), Handbook of Physiology; Section 7: The Endocrine System; Volume IV: Coping with the Environment: Neural and Endocrine Mechanisms. Oxford University Press pp. 517-532.

Sinha, R., 2008. Chronic stress, drug use and vulnerability to addiction. Ann. N. Y. Acad. Sci. 1141, 105-130. http://dx.doi.org/10.1196/annals.1441.030.

Sorregotti, T., Mendes-Gomes, J., Rico, J.L., Rodgers, R.J., Nunes-de-Souza, R.L., 2013. Ethopharmacological analysis of the open elevated plus-maze in mice. Behav. Brain Res. 246, 76-85. http://dx.doi.org/10.1016/j.bbr.2013.02.035.

Spruijt, B.M., Van Hooff, J.A.R.A.M., Gipsen, W.H., 1992. Ethology and neurobiology of grooming behavior. Physiol. Rev. 72, 825-852.

Tidey, J.W., Miczek, K.A., 1997. Acquisition id cocaine self-administration after social stress: role of accumbens dopamine. Psychopharmacology 130, 203-212.

Tinbergen, N., 1953. Social Behaviour in Animals: With Special Reference to Vertebrates. Wiley, Oxford, England.

Valzelli, L., 1985. Animal models of behavioral pathology and violent aggression. Methods Find. Exp. Clin. Pharmacol. 7, 189-193.

Van de Poll, N.E., De Jonge, F., Van Oyen, H.G., Van Pelt, J., 1982. Aggressive behaviour in rats: effects of winning or losing on subsequent aggressive interactions. Behav. Processes 7, 143-155. http://dx.doi.org/10.1016/0376-6357(82)90023-7.

Van Erp, A.M.M., Kruk, M.R., Meelis, W., Willekens-Bramer, D.C., 1994. Effect of environmental stressors on time course, variability and form of self-grooming in the rat: handling, social contact, defeat, novelty, restraint and fur moistening. Behav. Brain Res. 65, 47-55. http://dx.doi.org/10.1016/0166-4328(94)90072-8.

Yang, M., Augustsson, H., Markham, C.M., Hubbard, D.T., Webster, D., Wall, P.M., Blanchard, R.J., Blanchard, D.C., 2004. The rat exposure test: a model of mouse defensive behaviors. Physiol. Behav. 81, 465-473. http://dx.doi.org/10.1016/j. physbeh.2004.02.010.

Yoshimura, H., Kimura, N., 1991. Ethopharmacology of copulatory disorder induced by chronic social conflict in male mice. Neurosci. Biobehav. Rev. 15, 497-500. http:// dx.doi.org/10.1016/S0149-7634(05)80138-1. 